\title{
Improvement of Alignment Accuracy in Electron Tomography
}

\author{
Hyeong-Tae Jou*, Sujeong Lee ${ }^{1}$, Han-Joon Kim \\ Maritime Security Center, Korea Institute of Ocean Science \& Technology, Ansan 426-744, Korea \\ ${ }^{1}$ Mineral Resources Research Division, Korea Institute of Geoscience and Mineral Resources, Daejeon 305-350, Korea
}

\author{
*Correspondence to: \\ Jou HT, \\ Tel: +82-31-400-6278 \\ Fax: +82-31-408-5822 \\ E-mail: htjou@kiost.ac \\ Received January 22, 2013 \\ Revised March 6, 2013 \\ Accepted March 12, 2013
}

\begin{abstract}
We developed an improved method for tilt series alignment with fiducial markers in electron tomography. Based on previous works regarding alignment, we adapted the Levenberg-Marquardt method to solve the nonlinear least squares problem by incorporating a new formula for the alignment model. We also suggested a new method to estimate the initial value for inversion with higher accuracy. The proposed approach was applied to geopolymers. A better alignment of the tilt series was achieved than that by IMOD S/W. The initial value estimation provided both stability and a good rate of convergence since the new method uses all marker positions, including those partly covering the tilt images.
\end{abstract}

Key Words: Electron tomography, Geopolymer, Alignment, Fiducial marker

\section{INTRODUCTION}

Electron tomography is a method for analyzing a material's three-dimensional structure; its general resolution is between that of probe tomography (atomic resolution) and X-ray tomography ( $\mu \mathrm{m}$ resolution) (Ziese et al., 2004). Electron tomography has been widely applied in structural biology and material science. Recently, it broadens its application to medical science (e.g., Lee et al., 2005; Kim et al., 2012). Developments in the electron tomography technique have improved the resolution, which was about $1 \mathrm{~nm}$ in 2004, to 2.4 $\AA$ in research on three-dimensional structures using $10 \mathrm{~nm}$ gold nanoparticles (Midgley \& Dunin-Borkowski, 2009; Scott et al., 2012). Electron tomography is performed in four steps: (1) acquisition of 2-D transmission electron microscopy (TEM) projection images through continuous tilting of a sample, (2) alignment of acquired images, (3) reconstruction of 3-D tomogram, and (4) final visualization (De Rosier \& Klug, 1968).

Electron tomography is sensitive to the accuracy with which to obtain image data (Jou et al., 2008). Therefore, one of the most important steps in electron tomography is to align the
TEM images in order. Since independent two-dimensional computation is continuously used for three-dimensional restructuring owing to the computation time and memory constraints of current computers, reliable results cannot be obtained if the images are not all aligned accurately.

There have been many studies on image alignment; for effective alignment, fiducial markers made of gold nanoparticles are used if possible. Luther et al. (1988) proposed a convenient method where many variables can be variously applied; their method has been used for IMOD S/W (Kremer et al., 1996) and other applications. However, a drawback is that the fiducial markers should be present in every image. The fiducial markers can be observed even at high angles if the biological sample is thin or composed of light elements such as carbon; if the sample is thick or composed of heavy elements, the fiducial markers cannot be distinguished at high angles. In order to resolve this problem, Jing and Sachs (1991), Penczek et al. (1995), and others have proposed various methods that alter the least-squares method. For efficient analysis of the nonlinear least-squares method, Diez et al. (2006) combined Newton's method and the conjugate gradient method.

This research was supported by the Korea Institute of Ocean Science and Technology (Grant No. PE98977) and Ministry of the Environment's Waste Metal and Useful Resource Recycling Technology Development Project (Project No. GT-11-C-01-280-0) of the Global Top Environmental Technology Development Agency.

@ This is an open-access article distributed under the terms of the Creative Commons Attribution Non-Commercial License (http://creativecommons.org/licenses/by-nc/3.0) which permits unrestricted noncommercial use, distribution, and reproduction in any medium, provided the original work is properly cited.

Copyrights @ 2013 by Korean Society of Microscopy 
The objective of the present research is to develop several approaches to overcome the constraints of the methods that use fiducial markers to align images. We propose a modified model formula for image alignment and a nonlinear inverse formula that can deal with cases where a sufficient number of fiducial markers cannot be selected. The LevenbergMarquardt method (Levenberg, 1944; Marquardt, 1963), which is widely used in the geophysics, was used for mathematical formulation. An effective initial value selection method that can increase the stability and efficiency of convergence is also proposed. The feasibility of the proposed method was verified by tomography of a geopolymer sample.

\section{MATERIALS AND METHODS}

\section{Model Formula}

In order to align the images using markers, a mathematical model is first required to determine how the real location of the marker in three dimensions is mapped in each projected image. Various model formulas have been proposed by the previous studies noted in the introduction. In this study, we examine the formulas proposed by Luther et al. (1988) and Diez et al. (2006) that are widely used.

Luther et al. (1988) proposed the following model equation:

$$
P_{j}^{i}=S^{i} P A^{i} M^{i} y_{j}+d^{i}
$$

where $P_{j}^{i}\left(j=1, \cdots, n_{p}\right)$ represents the two-dimensional coordinates of the $i$-th image of the $j$-th marker from the $n_{p}$ number of markers; $y_{j}$ represents the $j$-th marker's real location coordinates in three dimensions; and $M^{i}$ is the $3 \times 3$ diagonal matrix, where its elements $m_{x}^{i}, m_{y}^{i}$, and $m_{z}^{i}$ represent the scale factors in the $\mathrm{x}, \mathrm{y}$, and $\mathrm{z}$ directions, respectively. $A^{i}$ is the $3 \times 3$ rotation matrix that defines the tilted degree of the sample; only the $y$-axis tilt is considered here. $P$ is the projection matrix that transforms the three-dimensional sample into a two-dimensional projection image, and $S^{i}$ is the two-dimensional rotation matrix of the $i$-th image. $d^{i}$ is the two-dimensional parallel shift vector of the $i$-th image.

Diez et al. (2006) proposed the following formula:

$$
\begin{aligned}
& s^{(j)} \hat{\mathrm{R}}\left(\alpha^{(j)}\right) \mathrm{P}_{\mathrm{z}} \mathrm{R}_{x}(-\phi) \mathrm{R}_{y}\left(\theta^{(j)}\right) \mathrm{R}_{x}(\phi)\left(\begin{array}{l}
x^{(i)} \\
y^{(i)} \\
z^{(i)}
\end{array}\right)=\left(\begin{array}{l}
p_{x}^{(i, j)} \\
p_{y}^{(i, j)}
\end{array}\right), \\
& \mathrm{R}_{x}(\phi)=\left(\begin{array}{ccc}
1 & 0 & 0 \\
0 & \cos (\phi) & \sin (\phi) \\
0 & -\sin (\phi) & \cos (\phi)
\end{array}\right)
\end{aligned}
$$

with,

$$
\begin{aligned}
& \mathrm{R}_{y}\left(\theta^{(j)}\right)=\left(\begin{array}{ccc}
\cos \left(\theta^{(j)}\right) & 0 & \sin \left(\theta^{(j)}\right) \\
0 & 1 & 0 \\
-\sin \left(\theta^{(j)}\right) & 0 & \cos \left(\theta^{(j)}\right)
\end{array}\right) \\
& \mathrm{P}_{\mathrm{z}}=\left(\begin{array}{lll}
1 & 0 & 0 \\
0 & 1 & 0
\end{array}\right), \text { and } \widehat{\mathrm{R}}\left(\alpha^{(j)}\right)=\left(\begin{array}{cc}
\cos \left(\alpha^{(j)}\right) & \sin \left(\alpha^{(j)}\right) \\
-\sin \left(\alpha^{(j)}\right) & \cos \left(\alpha^{(j)}\right)
\end{array}\right)
\end{aligned}
$$

$\left(x^{(i)}, y^{(i)}, z^{(i)}\right)$ is the $i$-th marker's location, and $\phi$ is the declination formed by the tilt axis and $x-y$ plane. $P_{z}$ is the $z$ direction's projection matrix, $\theta^{(j)}$ is the tilt angle, $\alpha^{(j)}$ is the rotation angle on the projection plane, and $s^{(j)}$ is the scale of the $j$-th image. $\left(p_{x}^{(i, j)}, p_{y}^{(i, j)}\right)$ represents the $i$-th marker's twodimensional location of the $j$-th image.

The two above formulas both contain the angle of rotation about the y-axis, scale, two-dimensional projection, and two-dimensional rotation, but they differ in several ways. In Luther et al.'s (1988) equation, three independent scale coefficients in the $\mathrm{x}, \mathrm{y}$, and $\mathrm{z}$ directions are given, whereas in Diez et al.'s (2006) equation, only one scale is applied after the projection. In particular, the latter adds a deviation term under the assumption that the tilt axis is not perpendicular to the $z$-axis due to equipment problems or experimental error. Furthermore, Diez et al. omit the parallel shift term, which may have been a typographical error or was already considered when the electron microscope image was obtained. Luther et al.'s (1988) method of using independent scales in the $\mathrm{x}, \mathrm{y}$, and $\mathrm{z}$ directions increases the flexibility for reducing various errors. Nevertheless, their results show some differences from those of an actual experiment, and it is realistic to use only one scale to compensate for the magnification error in each image after projection onto a twodimensional plane (Jing \& Sachs, 1991; Penczek et al., 1995). The deviation term added by Diez et al. (2006) can be useful for measuring the error that occurs in the actual experiment. However, the value cannot be used in two-dimensional tomography computation; in the case of the tomography goniometer, the term can be omitted because it is designed to minimize the deviation. The parallel shift term that is omitted in equation (2) must be included in the usual image alignment procedure for tomography.

Based on the above background, the following model equation was developed in this study:

$$
s^{(j)} \hat{\mathrm{R}}\left(\alpha^{(j)}\right) \mathrm{P}_{z} \mathrm{R}_{y}\left(\theta^{(j)}\right)\left(\begin{array}{c}
x^{(i)} \\
y^{(i)} \\
z^{(i)}
\end{array}\right)+\left(\begin{array}{l}
d_{x}^{(j)} \\
d_{y}^{(j)}
\end{array}\right)=\left(\begin{array}{c}
p_{x}^{(i, j)} \\
p_{y}^{(i, j)}
\end{array}\right)
$$

where every term is the same as those used in equation (2), except that only $\left(d_{x}^{(j)}, d_{y}^{(j)}\right)$ represents the $j$-th image's two- 
dimensional parallel shift distance. The tilt angle $\theta^{(j)}$, the rotation angle $\alpha^{(j)}$, and the shift distance vector $\left(d_{x}^{(j)}, d_{y}^{(j)}\right)$ are illustrated in Fig. 1A and B, respectively.

\section{Nonlinear Least-Squares Method}

Using equation (3) as the premise, the procedure of aligning images becomes a problem of finding those variables such as each image's scale $s^{(j)}$, the rotation angle on the plane $\alpha^{(j)}$, the tilt angle $\theta^{(j)}$, the marker's three-dimensional location $\left(x^{(i)}\right.$, $\left.y^{(i)}, z^{(i)}\right)$, and the parallel shift distance $\left(d_{x}^{(j)}, d_{y}^{(j)}\right)$ that minimize the difference between the marker's coordinate $\left(\hat{p}_{x}^{(i, j)}, \hat{p}_{y}^{(i, j)}\right)$ and equation (3)'s theory model value of $\left(p_{x}^{(i, j)}, p_{y}^{(i, j)}\right)$. Thus, the solution that minimizes the error $\mathrm{L}$ in the following root mean square (RMS) error needs to be solved.

$$
L=\sqrt{\sum^{N_{p}}\left(\left(\hat{p}_{x}^{(i, j)}-p_{x}^{(i, j)}\right)^{2}+\left(\hat{p}_{y}^{(i, j)}-p_{y}^{(i, j)}\right)^{2}\right)} /\left(2 N_{p}\right)
$$

Here, $N_{p}$ is the total number of selected markers used in the least-squares method.

The least-squares equation above is a typical nonlinear inverse problem where the unknowns have complicated relations. The previous studies used various methods to solve this type of nonlinear least-squares problem. In the least-squares the stability of convergence is very important.

Luther et al. (1988) did not choose a method that directly minimizes L, instead, used various conditions beforehand so that all the variables do not affect each other as much as possible. The most important condition of all in their method was that the marker used in the calculation should exist in each image. This condition makes the problem very simple and increases the stability of convergence (IMOD S/W also use this method). However, if the sample is too thick in practice or there is too much experimental noise, it becomes more difficult to distinguish the marker in the electron microscopy image at higher angles. Thus, it is difficult to secure this condition except for thin biological samples. If $\mathrm{L}$ in equation (4) is to be minimized directly, the constraints devised by Luther et al. (1988) are eliminated because it is possible to make $\mathrm{L}$ that is composed of only distinguishable markers in each image. The nonlinear least-squares method should minimize L. The Levenberg-Marquardt (L-M) method, which is widely used for nonlinear inverse operations in various disciplines such as geophysics, was used in the present study. The L-M method is a method known to provide stability and efficiency of convergence of two methods: (1) the gradient descent method, where convergence is slow but accurately approaches the solution, and (2) Newton's method, where convergence is fast but there is a risk of divergence.

The most difficult problems of using the L-M method to minimize $L$ in equation (4) are the dependency between the

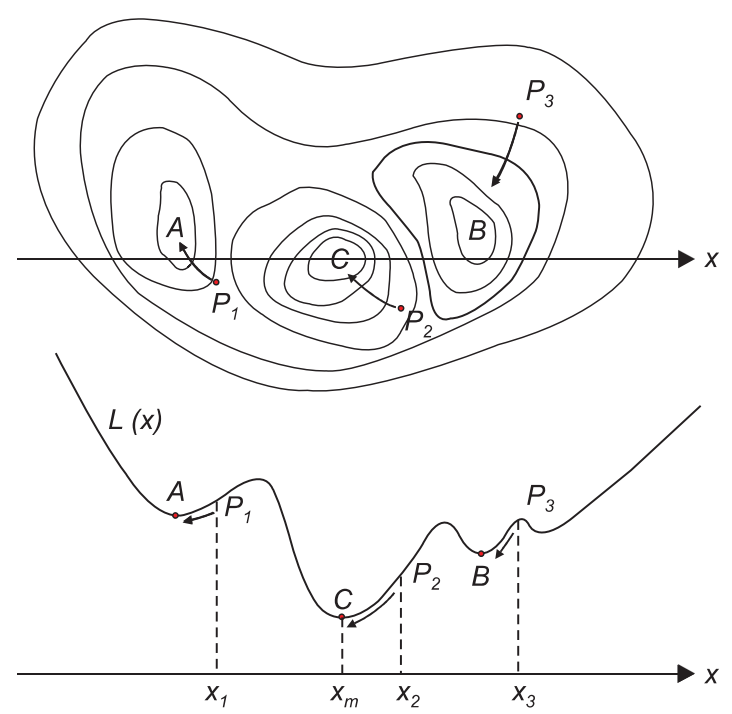

Fig. 2. Schematic presentation of local and true minima. A and B are local minima, and $\mathrm{C}$ is a true (or global) minimum.
A

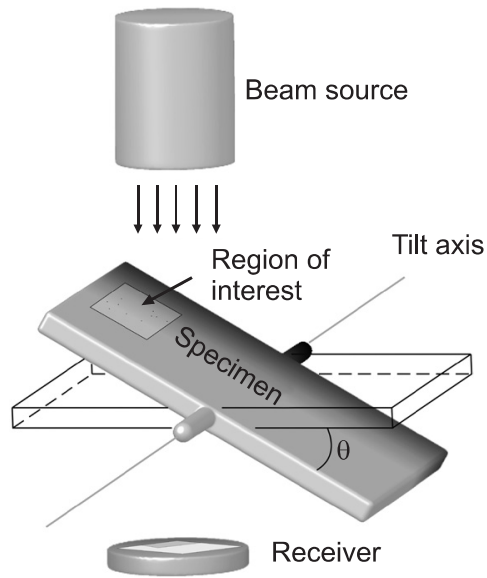

B

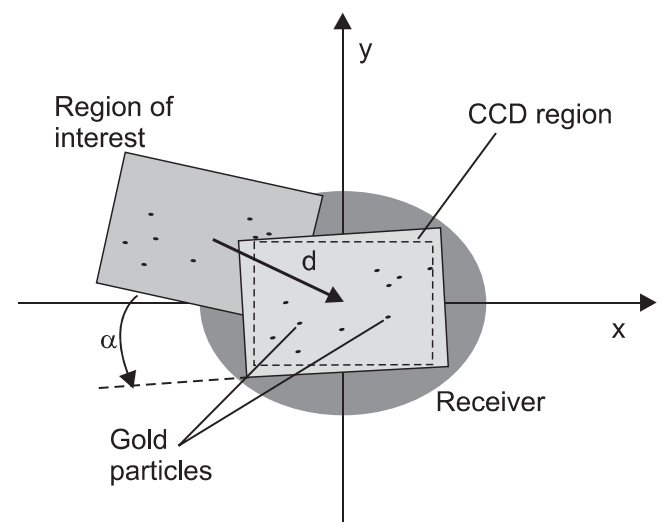

Fig. 1. Sketch of a specimen subjected to (A) tilting, and (B) rotation and parallel shift. $\theta^{(j)}$ is the tilt angle; $\alpha^{(j)}$ and $\mathrm{d}$ are the rotation angle and parallel displacement on the projection plane, respectively. 
variables and the initial value problem. Equation (3) actually has many minimum values because the parameters are closely related to each other; in this case, the initial value selection has a huge influence on the stability of convergence. With regard to the minimizing problem, the local minimum value's influence is schematically illustrated in Fig. 2. Three minimum values exist in the figure, and the true minimum value of these three is at $C\left(x=x_{m}\right)$. If one of the points in the figure is selected as the initial value, the convergence direction progresses in the direction that reduces the RMS error. If $P_{2}\left(x_{0}=x_{2}\right)$ is selected as the initial value, it converges to $C\left(x=x_{m}\right)$, which is the true minimum value. However, if $P_{1}\left(x_{0}=x_{1}\right)$ or $P_{3}\left(x_{0}=x_{3}\right)$ is selected as the initial value, it converges toward the local minimum such as $A$ and $B$ and cannot converge to the accurate minimum value $C$. In short, for a complicated nonlinear leastsquares method, the initial value selection is very important. In particular, if there are many unknown variables and the dependency between each variable is strong, then convergence cannot be obtained properly; measures to solve this are also needed.

In the above equation, let the total number of images be $N$, the total number of marker categories be $M$, and the total number of marker coordinates selected in the entire image be $N_{p}$. The given measurement is $2 N_{p}$ because there are $N_{p}$ number of $(x, y)$ coordinates. Since there are five variables for each image such as scale, rotation angle, tilt angle, and $\mathrm{x}$ and $y$ parallel shifts, the number of unknowns is $5 N$. Since the number of each marker's $(x, y, z)$ coordinate variables is $3 M$, the total number of unknowns is $5 N+3 M$. For example, 100 images and 30 marker categories have 590 unknown variables, so this problem would be difficult to solve because of the large inverse operations involved. Thus, in equation (3), since the tilt angle and various different variables are closely related, it is

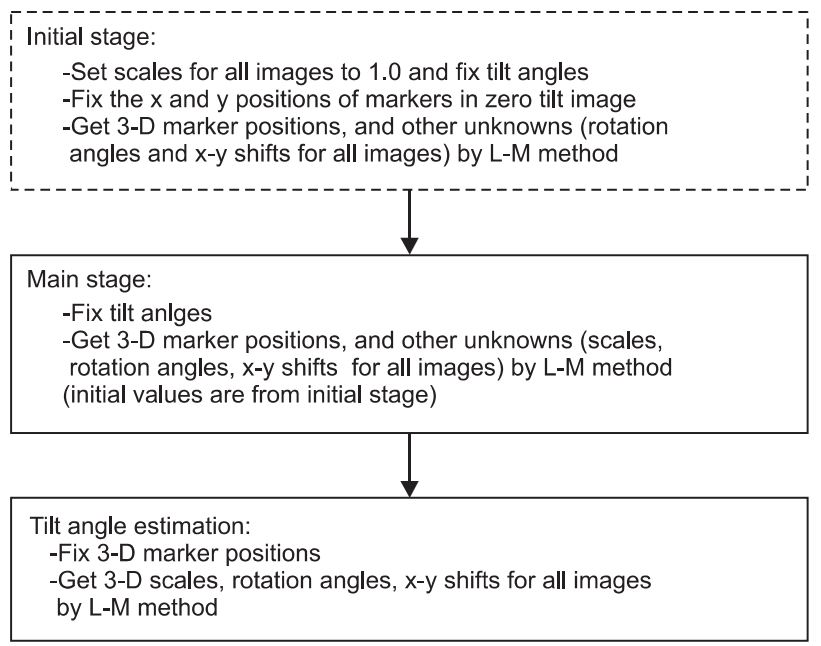

Fig. 3. Flowchart for the alignment process using the LevenbergMarquardt (L-M) method. highly probable that direct minimization of all the unknowns do not converge. To overcome this issue, the rotating angle is determined beforehand using existing information in the present study; the rest of the variables are determined using inverse operation formulas. During estimation, the rotation angle is then taken again. This procedure is shown as a flowchart in Fig. 3: a two-step inversion process (main stage in the figure) and tilt estimate.

To improve the efficiency of the inversion process, a new method was developed. The initial estimation stage improves the initial value input before the main inversion operation process is performed (Fig. 3). In the initial estimation stage, the number of variables with strong dependency is reduced to minimize the number of unknowns. In this case, the number of local minimum values decrease, and a stable solution can be found. The unknown values found in this initial stage are reused as initial values in the main inversion operation, which increases the number of unknowns. This improves the stability of conversion and the speed of computation as well.

\section{Test Data}

TEM images of geopolymers were used to test the proposed method. The objective of the test is to examine the pore characteristics of the geopolymer. TEM observations were performed using the FEI Tecnai G2 Spirit at an acceleration voltage of $120 \mathrm{kV}$. A total of 107 images at a resolution of $2,048 \times 2,048$ pixels were obtained at a magnification of $40,000 \times$ and at $1^{\circ}$ intervals in a tilt angle range of $-55^{\circ}$ to

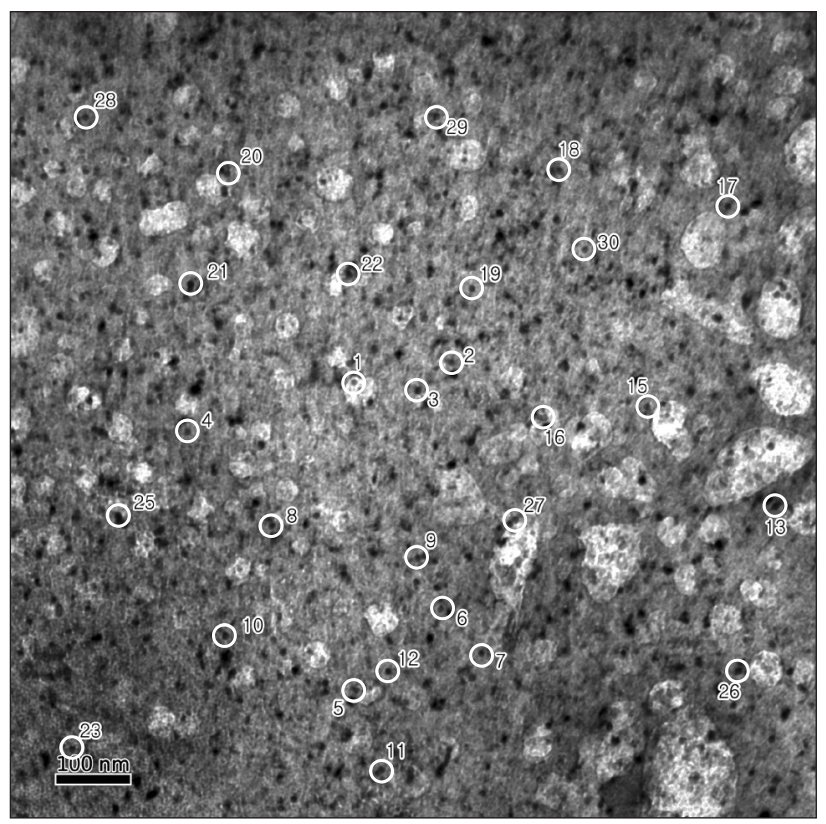

Fig. 4. Transmission electron microscopy bright-field micrograph of the geopolymer lamella. Gold fiducial markers and many small crystals were observed along with various-sized pores. White circles with numbers around them indicate selected markers and their ID numbers. 
$53^{\circ}$. The markers were gold nanoparticles with diameters of $10 \mathrm{~nm}$. Fig. 4 shows the image when tilted at $0^{\circ}$; the white circle represents the location of the marker labeled with the marker's ID number.

The circular white objects in the figure are the pores in the geopolymer, and the black objects are the diffraction effect of the gold nanoparticles and crystalline particles. Most of the samples were amorphous.

\section{RESULTS AND DISCUSSION}

When aligning the test data, it was observed that the crystalline materials' diffraction, as shown in Fig. 4, affected the tomography results as noise because the change in contrast occurred frequently depending on tilting. Since the markers are difficult to distinguish, aligning the images was also difficult. For all 30 markers, we attempted to determine their locations on all TEM tilted images; markers difficult to distinguish were not selected. In total, 2,900 marker locations were selected. Among them, only six common markers (numbered 2, 6, 7, 12, 19, and 20 in Fig. 4) existed in all of the images.

\section{IMOD S/W Results}

First, IMOD S/W, which is widely used in electron tomography, was used for image alignment. Since IMOD S/W is based on Luther et al.'s (1988) theory, only the markers existing in all of the images could be used. Thus, only the six markers $(2,6,7$, 12, 19, 206) in Fig. 4 were selected. Fig. 5 shows the markers' locations in IMOD S/W and the alignment procedure. During the alignment, the RMS error was found to be 0.839 , and it took about 8 seconds to solve.

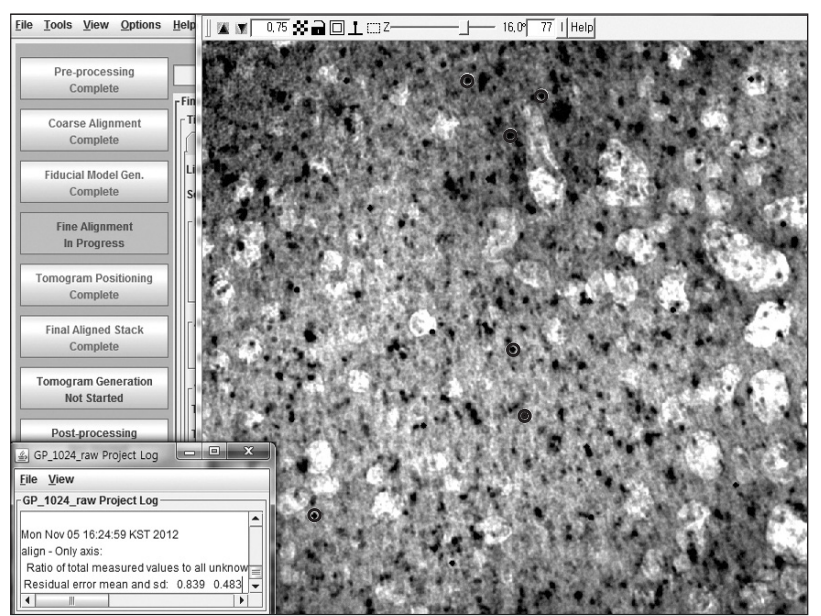

Fig. 5. Window display showing the alignment process in the IMOD S/ W. Circles in the transmission electron microscopy image indicate the markers used for alignment. The residual error mean or root mean square error is shown in the lower left corner.

\section{Solution Using the Levenberg-Marquardt Method}

Similar to IMOD S/W, six markers were selected and applied to the L-M method proposed in this study. Since there were 107 images in total and six types of markers, the total number of measurements was $107 \times 6 \times 2=1,284$. The total number of unknowns was $107 \times 5+6 \times 3=553$. Initially, for the basic L-M process, we reduced the number of unknowns to 533$107=425$ by directly using the values of the tilt angles. The correct tilt angles were estimated after inversion.

For the improved L-M method, the initial selection procedure was conducted before inversion. Several variables were fixed beforehand to reduce the number of unknowns. First, the image tilted at $0^{\circ}$ was projected assuming that the original sample was not modified through tilting, rotation, and parallel shifting. Then, the coordinate value selected for the image acquired at $0^{\circ}$ was fixed beforehand. Since the difference in magnification of each image was very small, all of the scales were fixed to 1.0. Through these two procedures, the total number of unknowns in the L-M method used for the initial value selection procedure was significantly reduced. In this example, the number of unknowns was decreased by 119 , and the total number of unknowns became $426-119=307$. At this stage, the L-M method was applied to estimate the values of the unknown variables, followed by the regular L-M procedure.

Table 1. Alignment result using Levenberg-Marquardt (L-M) method with only six markers

\begin{tabular}{|c|c|c|c|c|}
\hline & \multicolumn{4}{|c|}{ L-M method } \\
\hline & \multicolumn{2}{|c|}{ Without initial stage } & \multicolumn{2}{|c|}{ With initial stage } \\
\hline & $\begin{array}{l}\text { RMS residual } \\
\text { (pixel) }\end{array}$ & $\begin{array}{l}\text { CPU time } \\
\text { (s) }\end{array}$ & $\begin{array}{l}\text { RMS residual } \\
\text { (pixel) }\end{array}$ & $\begin{array}{l}\text { CPU time } \\
\text { (s) }\end{array}$ \\
\hline Initial stage & - & - & 1.508 & 2.95 \\
\hline Main stage & 0.723 & 11.51 & 0.723 & 6.21 \\
\hline Tilt estimate & 0.653 & 0.02 & 0.653 & 0.02 \\
\hline \multirow[t]{2}{*}{ Final result } & \multicolumn{2}{|c|}{ RMS residual $=0.653$ pixel } & \multicolumn{2}{|c|}{ RMS residual $=0.653$ pixel } \\
\hline & \multicolumn{2}{|c|}{ CPU time $=11.53 \mathrm{~s}$} & \multicolumn{2}{|c|}{$\mathrm{CPU}$ time $=9.18 \mathrm{~s}$} \\
\hline
\end{tabular}

Table 2. Alignment result using Levenberg-Marquardt (L-M) method with all 30 markers

\begin{tabular}{lccccc}
\hline & \multicolumn{4}{c}{ L-M method } \\
\cline { 2 - 3 } & \multicolumn{2}{c}{ Without initial stage } & & \multicolumn{2}{c}{ With initial stage } \\
\cline { 2 - 3 } \cline { 5 - 6 } & $\begin{array}{c}\text { RMS residual } \\
\text { (pixel) }\end{array}$ & $\begin{array}{c}\text { CPU time } \\
\text { (s) }\end{array}$ & & $\begin{array}{c}\text { RMS residual } \\
\text { (pixel) }\end{array}$ & $\begin{array}{c}\text { CPU time } \\
\text { (s) }\end{array}$ \\
\hline Initial stage & - & - & & 2.331 & 9.83 \\
Main stage & 0.913 & 78.55 & & 0.913 & 20.65 \\
Tilt estimate & 0.895 & 0.02 & & 0.895 & 0.02 \\
Final result & RMS residual $=0.895$ pixel & & \multicolumn{2}{c}{ RMS residual $=0.895$ pixel } \\
& \multicolumn{2}{c}{ CPU time $=78.57 \mathrm{~s}$} & & \multicolumn{2}{c}{ CPU time $=30.50 \mathrm{~s}$} \\
\hline
\end{tabular}



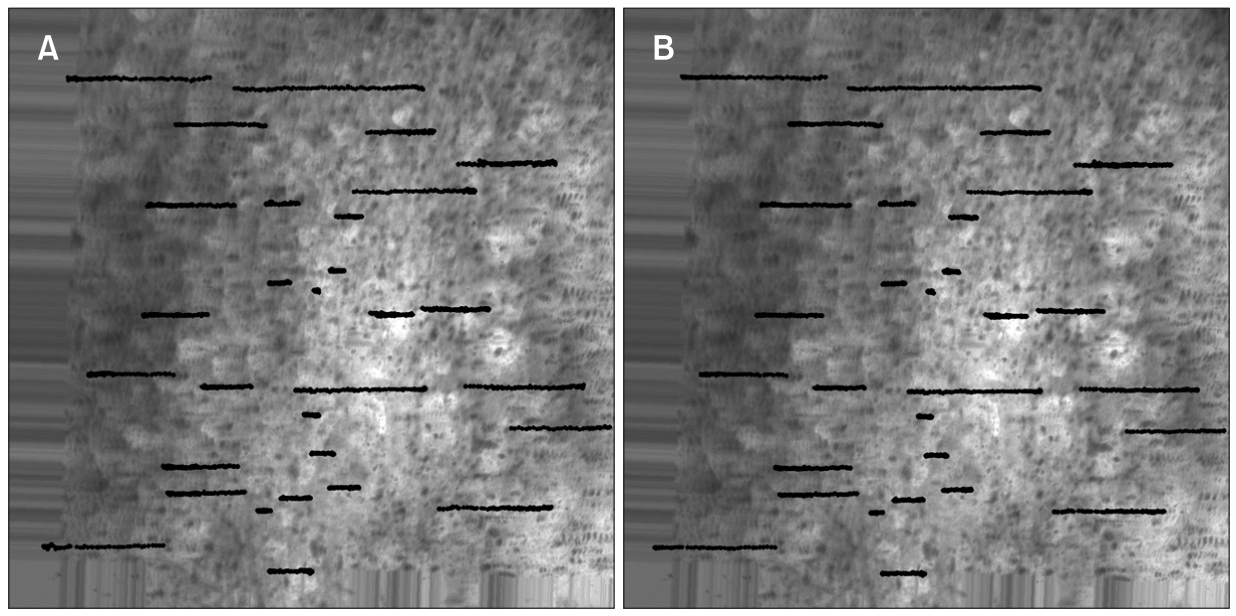

Fig. 6. Overlapped images of aligned tilt series (A) using only six markers and (B) using all markers. More horizontal marker trajectories mean better results.

Table 1 lists the image alignment results using the L-M method and compares the differences when the initial value estimate procedure was and was not performed. As shown in the results, the RMS error was reduced to 0.653 from IMOD S/W's 0.839 , but it took slightly longer to compute. The improved L-M method took less time to compute than the regular L-M method, but the difference was very small because there were a small number of unknown variables. For the L-M method, the RMS error was smaller than IMOD because of the algorithm characteristics and convergence conditions. The image alignment results were almost the same.

As noted above, the disadvantage of IMOD S/W is that it requires the markers to exist in all images. The results of the inversion performed on all of the selected points were considered to verify the efficiency of the inversion method used in this research.

All 30 of the marker categories were selected; all of more than 2,900 marker coordinates selected from the entire image were used in the L-M method. Table 2 lists the inversion results. The results show that the RMS error was slightly higher than the case of using six markers, as shown in Table 1, and it took fairly long to compute. Compared to the basic L-M method, the initial value improved the L-M method's convergence speed by 2.5 times.

\section{Comparison of Tomography Results}

We then compared the image alignment results of using only the six markers existing in all of the images, as in IMOD S/ $\mathrm{W}$, and using all of the 30 markers randomly present in the images.

One way to determine the accuracy of image alignment is to overlap each aligned image and then observe changes in prominent markers. If the images are aligned, the markers on the overlapped images move parallel along the $\mathrm{x}$-axis (i.e., the $y$-value remains the same). However, it is difficult

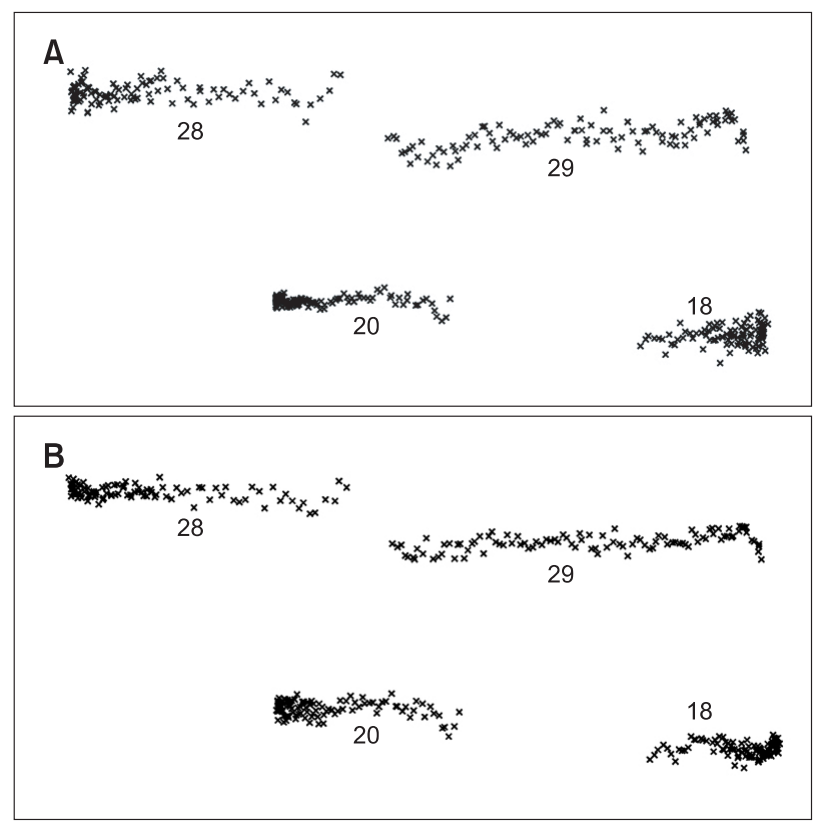

Fig. 7. Plots of the marker positions 18, 20, 28, and 29 in Fig. 6 (A) using only six markers and (B) using all markers. Vertical variations are exaggerated three times for display.

to use this method because the markers are not clearly distinct in the TEM images (Fig. 4) used in the experiment. To solve this problem, points were artificially added onto the markers' coordinates, which were selected among all images, to distinguish them from each other; the aligned images were then overlapped. Fig. 6 shows the overlapped images to compare the results of using only six markers and that of using all 30 markers.

Fig. $6 \mathrm{~A}$ and $\mathrm{B}$ show the results of alignment using 6 and 30 markers, respectively. In both cases, alignment was successfully achieved. However, there is no clear difference between them. For a more detailed comparison, the coordinates of the points 

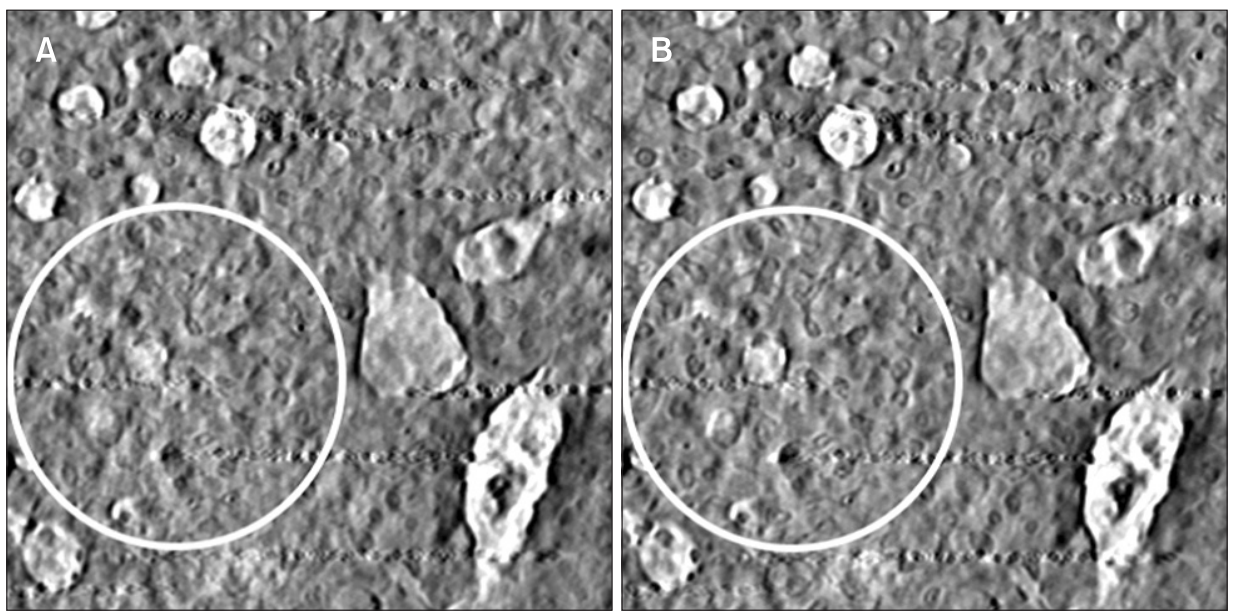

Fig. 8. Comparison of tomographic reconstructions in the two cases. Each z-slice is from the tomograms (A) using six markers and (B) using 30 markers. Circles highlight the improvement in the results.

aligned on the top part of Fig. 6 were drawn into symbols shown in Fig. 7. To make the comparison more convenient, the change in y-axis was exaggerated threefold. In Fig. 7, the changes in markers 18,28 , and 29 showed better results when 30 markers were used. Marker 20 showed better results when six markers were used. This may be because marker 20 was the one directly used when the six-marker alignment was performed.

To determine the influence of the two-image alignment results on tomography, tomography using the filtered backprojection method was performed on each aligned image. Fig. 8 shows the extracted the $x-y$ section from each final tomogram to compare the results. In Fig. 8, the results look similar; however, the tomogram obtained using 30 markers (Fig. 8B) shows an improved and clearer image than the section obtained using six markers (Fig. 8A), as highlighted by a circle.

Very precise image alignment is required to obtain a higherresolution tomography result. The improved L-M method proposed above makes it possible to effectively determine the precise image alignment. The approach proposed in this study would be very useful when it is difficult to discriminate the markers, especially when tomography is to be applied to TEM images with thick samples and in the presence of noise.

\section{SUMMARY}

We proposed a new model formula using the L-M method and an initial valuable selection procedure to improve the efficiency of image alignment, which is an essential stage in electron tomography. The conclusions obtained from tests on geopolymer TEM images to verify the performance can be summarized as follows:

1. The newly proposed model formula and the minimizing method have the advantages of using all of the markers selected from the random images and find a stable solution to inversion.

2. The newly proposed initial value selection method greatly reduces the computation time of the original L-M method.

3. The image alignment method proposed in this paper is useful for the cases where the sample is thick or it is difficult to select all of markers in the entire image due to significant noise.

\section{REFERENCES}

De Rosier D J and Klug A (1968) Reconstruction of three dimensional structures from electron micrographs. Nature 217, 130-134.

Diez D C, Seybert A, and Frangakis A S (2006) Tilt-series and electron microscope alignment for the correction of the non-perpendicularity of beam and tilt-axis. Ultramicroscopy 154, 195-205.

Jing Z and Sachs F (1991) Alignment of tomographic projections using an incomplete set of fiducial markers. Ultramicroscopy 35, 37-43.

Jou H T, Lee S, Kim Y J, and Suk B C (2008) Model simulation for assessment of image acquisition errors affecting electron tomography. Korean J. Microscopy 38, 51-61.
Kim M J, Huh Y H, Choi K J, Jun S, Je A R, Chae H, Lee C, and Kweon H $S$ (2012) Ultra-structural abnormalities in APP/PSEN1 transgenic mouse brain as the Alzheimer's disease model. Appl. Microsc. 42 , 179-185.

Kremer J R, Mastronarde D N, and McIntosh J R (1996) Computer visualization of three-dimensional image data using IMOD. J. Struct. Biology 116, 71-76.

Lee K J, Kweon H S, Kang J S, and Rhyu I J (2005) 3-dimensional reconstruction of parallel fiber-Purkinje cell synapses using highvoltage electron microscopy. Korean J. Microscopy 35, 31-39. 
Jou HT et al.

Levenberg K (1944) A method for the solution of certain problems in least squares. Quart. Appl. Math. 2, 164-168.

Luther P K, Lawrence M C, and Crowther R A (1988) A method for monitoring the collapse of plastic sections as a function of electron dose. Ultramicroscopy 24, 7-18.

Marquardt D (1963) An algorithm for least-squares estimation of nonlinear parameters. SIAM J. Appl. Math. 11, 431-441.

Midgley P A and Dunin-Borkowski R E (2009) Electron tomography and holography in materials science. Nature Mater. 8, 271-280.
Penczek P, Marko M, Buttle K, and Frank J (1995) Double-tilt electron tomography. Ultramicroscopy 60, 393-410.

Scott M C, Chen C-C, Mecklenburg M, Zhu C, Xu R, Ercius P, Dahmen U, Regan B C, and Miao J (2012) Electron tomography at 2.4-ångström resolution. Nature 483, 444-447.

Ziese U, Jong K P de, and Koster A J (2004) Electron tomography: a tool for 3D structural probing of heterogeneous catalysts at the nanometer scale. App. Catal. A-Gen. 260, 71-74. 